\title{
Tunneling of Optical Beams through Inhomogeneity of a Refractive Index
}

\author{
A. A. Kalinovich ${ }^{a}$, V. E. Lobanov ${ }^{a}$, A. P. Sukhorukov ${ }^{a}$, and A. L. Tolstik ${ }^{b}$ \\ ${ }^{a}$ Moscow State University, Moscow, 119991 Russia \\ ${ }^{b}$ Belarus State University, Minsk, Belarus
}

\begin{abstract}
We consider the effect of tunneling of optical beams through a narrow induced inhomogeneity in a refractive index. It is shown that under the condition of total internal reflection from the induced channel, part of the signal beam leaks if the channel is narrow. Dependence of the pump beam width at which the tunneling of half signal power occurs is found as a function of the pump intensity and the angle of beam crossing.
\end{abstract}

DOI: $10.3103 / \mathrm{S} 1062873810120221$

\section{INTRODUCTION}

The nonlinear reflection of optical beams from inhomogeneities induced by other optical beams (i.e., the light reflection of light) is currently of great interest of researchers [1-3]. A high-power pump wave generates negative inhomogeneities in the refractive index for another wave in defocusing media. The minimum of the refractive index is related to the maximum of pump intensity. The signal beam is refracted upon propagation through an inhomogeneous channel. If the angle of beam crossing is narrow, the trajectory is so distorted that total internal reflection takes place.

This phenomenon has been studied well upon cascade three-frequency interaction in quadratic media [4], photorefractive media [5], and media with thermal defocusing nonlinearity [6].

The greater the pump intensity, the higher the signal beam's tilt angle; to achieve the condition of nonlinear total reflection, we therefore require a considerable power density of the pump beam. The pump beam is thus focused, reflection occurs near the waist, and the width of the inhomogeneity in the waist can be narrow. In this work, we considered the impact of pump beam width on the reflection dynamics. The dependence of a portion of the tunneling wave energy on the nonlinear channel width (essentially the width of the pump beam) was found by means of numerical simulation. We concluded that the propagation of part of a signal beam part through the pump beam is analogous to the quantum tunneling effect. It is known from quantum mechanics that the dependence of the transmission factor upon the tunneling of a particle through a rectangular potential barrier is [7]:

$$
T \sim \exp \left(-\frac{2}{\hbar} \sqrt{2 m(u-E)} L\right)
$$

where $\hbar$ is the Planck constant, $m$ is the particle mass, $u$ is the potential barrier value, $E$ is the particle energy, and $L$ is the potential barrier width. The value of the transmission factor is exponentially reduced upon a barrier increase.

\section{BEAM INTERACTION IN A QUADRATIC MEDIUM}

We first bconsider parametrical beam reflection upon three-frequency interaction. The system of equations for envelopes $A_{j}(j=1,2,3)$ can be written in the following form:

$$
\begin{gathered}
\frac{\partial A_{1}}{\partial z}+i D_{1} \Delta_{\perp} A_{1}=-i \gamma_{1} A_{3} A_{2}^{*}, \\
\frac{\partial A_{2}}{\partial z}+i D_{2} \Delta_{\perp} A_{2}=-i \gamma_{2} A_{3} A_{1}^{*}, \\
\frac{\partial A_{3}}{\partial z}+i D_{3} \Delta_{\perp} A_{3}=i \Delta k_{m} A_{3}-i \gamma_{3} A_{1} A_{2},
\end{gathered}
$$

where $D_{j}=\left(2 k_{j}\right)^{-1}$ is a diffraction coefficient, $\gamma_{j}=$ $2 \pi \mathbf{e}_{1} \bar{\chi}^{(2)} \mathbf{e}_{2} \mathbf{e}_{3} \omega_{j} /\left(c n_{j}\right)$ is a nonlinearity coefficient, $\omega_{j}$ is the carrier frequency, $\hat{\chi}^{(2)}$ is the tensor of quadratic nonlinear susceptibility, $n_{j}$ is the linear refractive index, $c$ is the speed of light, and $\Delta k_{m}=k_{1}+k_{2}-k_{3}$ is the dispersive detuning of wave vectors. The initial conditions are written as

$$
\begin{gathered}
A_{1}(z=0)=E_{1} \exp \left(-x^{2} / w_{1}^{2}\right), \\
A_{2}(z=0)=E_{2} \exp \left[-(x-d)^{2} / w_{2}^{2}+i \theta x\right]
\end{gathered}
$$


where $E_{1,2}$ is the beam amplitude, $w_{1,2}$ is the beam width, $d$ is the initial displacement, and $\theta$ is the tilt angle of a signal beam.

System of equations (2) is numerically solved for different values of a pump beam width $w_{1}$. We investigated the case of nonsynchronous cascade interaction upon the large detuning of wave vectors $\Delta k \gg \gamma_{3} E_{1}$ under the conditions of the given pump field $E_{2} \ll E_{1}$. The dynamics of the propagation of an inclined signal beam moving toward the pump beam was considered. Due to the interaction, one part of the signal beam is reflected while the other part passes through the inhomogeneity induced by the pump. The modeling results are given in Fig. 1a. It can be seen that reflection coefficient $R$ is reduced and transmission factor $T$ is increased from zero as the pump width declines. Since part of energy arrives at the third beam at frequency $\omega_{3}$, the sum of coefficients $R$ and $T$ is less than unity. Introducing the positive detuning of wave vectors $\Delta k=10$ slightly increases beam propagation, but amplifies the transfer to the third frequency. Since the effect of tunneling is mixed with the effect of energy transfer to the third frequency, this is inconvenient for analysis.

\section{BEAM TUNNELING IN A PHOTOREACTIVE CRYSTAL}

Effective two-frequency interaction can be attained in a medium with photoreactive nonlinearity. The equations for envelopes $A_{j}(j=1,2)$ have following form:

$$
\begin{aligned}
& \frac{\partial A_{1}}{\partial z}+i D_{1} \Delta_{\perp} A_{1}=i \gamma_{1} \frac{A_{1}}{1+\alpha\left(\left|A_{1}\right|^{2}+\left|A_{2}\right|^{2}\right)} \\
& \frac{\partial A_{2}}{\partial z}+i D_{2} \Delta_{\perp} A_{2}=i \gamma_{2} \frac{A_{2}}{1+\alpha\left(\left|A_{1}\right|^{2}+\left|A_{2}\right|^{2}\right)}
\end{aligned}
$$

where $D_{j}$ is a diffraction coefficient, $\gamma_{j}$ is a nonlinearity coefficient, and $\alpha$ is a parameter describing nonlinearity saturation. Equations (4) were solved numerically. The dependences of reflection coefficient $R$ and transmission factor $T$ are presented in Fig. 1b. A portion of the energy propagates through the induced nonlinearity at narrow widths of the pump beam, and the reflection coefficient is reduced and the transmission factor is increased. The effect is analogous to quantum tunneling through a potential barrier of finite width.

We now consider the dependence of the pump beam width at which a half beam propagates $(T=0.5)$, on the pump intensity value and the signal beam's angle of incidence. The results from numerical simulation of the dependence of the pump beam's critical width on the intensity at two different fixed angles of incidence are represented in Fig. 2a by
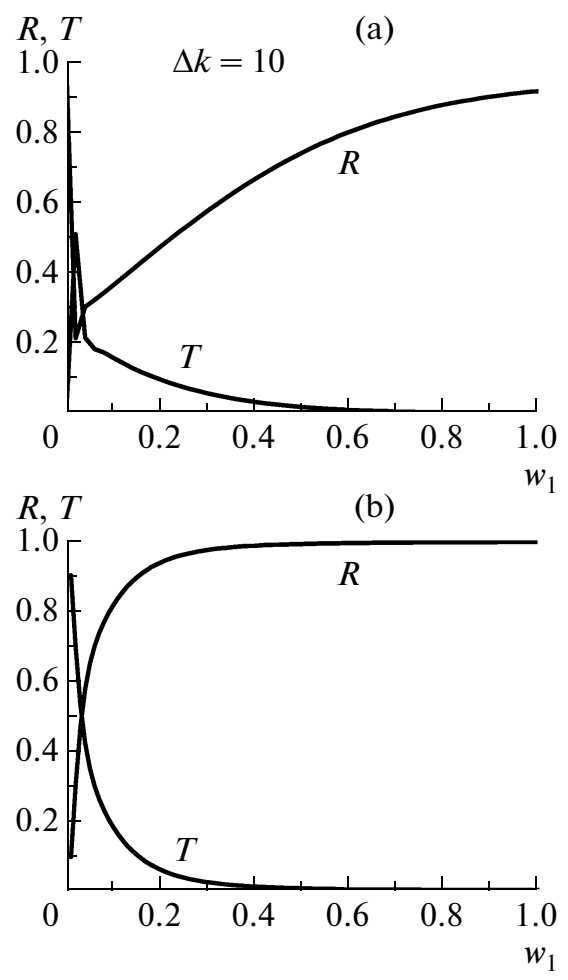

Fig. 1. Dependences of reflection coefficient $R$ and transmission factor $T$ on pump beam width $w_{1}$ upon three-frequency interaction in a quadratic nonlinear crystal (a) and in photorefractive crystal (b).

points. The critical value of pump beam width $w_{\text {lcr }}$ increases as the intensity $A_{1}^{2}$ declines, due to a weakening of refraction in the inhomogeneity and a reduction in the critical tilt angle of the signal beam, which cannot be exceeded if total reflection is to be attained [4].The signal beam thus passes through the pump beam easily and the critical value of the pump beam width rises freely.

In analogy to Eq. (1), we may assume that the transmission factor can be described by the formula $T \sim \exp \left(-\sqrt{A_{1}^{2}-A_{1 \mathrm{cr}}^{2}} w_{1}\right)$ upon the interaction of optical beams. It follows that for fixed tunneling level $T$, a simple dependence of the critical pump beam width on pump intensity can be obtained in the form

$$
w_{1 \mathrm{cr}} \sim \ln T / \sqrt{A_{1}^{2}-A_{1 \mathrm{cr}}^{2}} .
$$

The numerical simulation data of Eqs. (4) were approximated by the curves of type (5) shown in Fig. 2a by solid lines. There is good agreement between the tunneling theory of optical beams in nonlinear media and the well-known representations of quantum mechanics.

We also obtained the dependence of the pump beam width on the tilt angle of the signal beam relative 

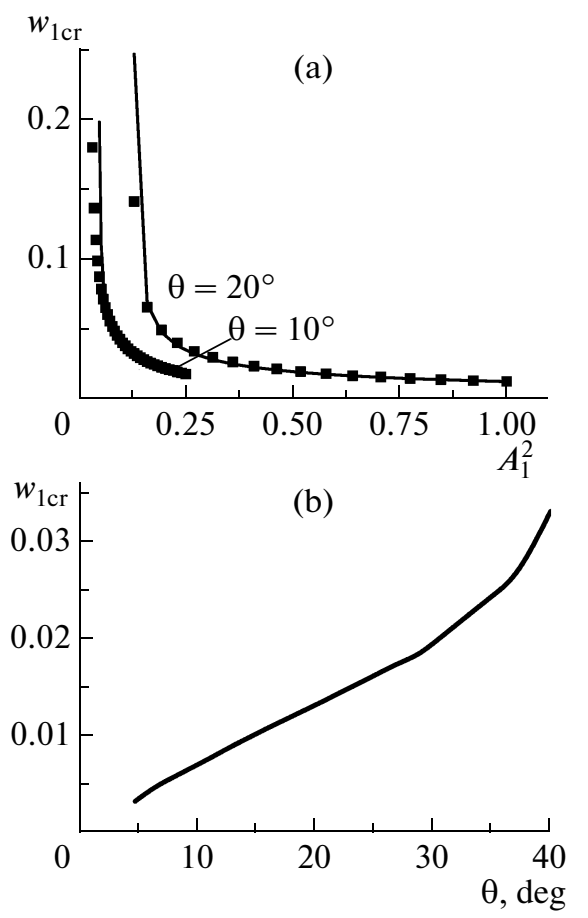

Fig. 2. Dependences of the critical pump beam width $w_{1 \mathrm{cr}}$, at which half the power of the signal beam is tunneled on pump intensity (a) and the tilt angle of the signal beam (b).

to the pump beam. We can see that the critical value of the pump beam width increases as the tilt angle rises (Fig. 2b), confirming the theory of total reflection in photorefractive crystals [5].

\section{CONCLUSIONS}

The tunneling of optical beams through an induced inhomogeneity was investigated. It was shown that part of a signal beam can propagate through a narrow induced inhomogeneity despite the conditions of total internal reflection. The dependences of the pump beam width needed for the reflection of half the signal beam's energy on the pump intensity and the tilt angle of the signal beam were found for photorefractive media.

\section{ACKNOWLEDGMENTS}

This work was supported in part by the Russian Foundation for Basic Research, project nos. 08-0200717, 09-02-01028, 10-02-90010-Bel, and the Belarus Republic's Foundation for Fundamental Research, project F10R-070.

\section{REFERENCES}

1. Mingaleev, S. and Kivshar, Yu.S., Opt. Photon News, 2002, no. 7 , p. 48.

2. Kivshar, Yu.S. and Stegman, G.I., Opt. Photon News, 2002, no. 2, p. 59.

3. Pertsch, T., Peschel, U., and Lederer, F., Opt. Lett., 2003, vol. 28, no. 2, p. 102.

4. Lobanov, V.E. and Sukhorukov, A.P., Izv. Akad. Nauk. Ser. Fiz., 2005, vol. 69, no. 12, p. 1775.

5. Kabakova, I.V. and Sukhorukov, A.P., Izv. Akad. Nauk. Ser. Fiz., 2006, vol. 70, no. 12, p. 1752.

6. Lobanov, V.E., Kalinovich, A.A., Sukhorukov, A.P., et al., Laser Phys., 2009, vol. 19, p. 1112.

7. Blokhintsev, D.I., Osnovy kvantovoi mekhaniki (Foundations of Quantum Mechanics), St. Petersburg: Lan', 2004. 